\title{
Reactive plasma cleaning and restoration of transition metal dichalcogenide monolayers
}

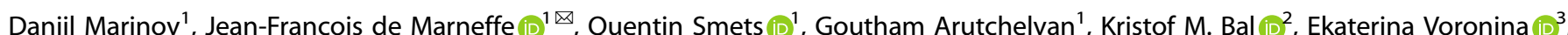
Tatyana Rakhimova ${ }^{3}$, Yuri Mankelevich $\mathbb{1 D}^{3}$, Salim El Kazzi ${ }^{1}$, Ankit Nalin Mehta ${ }^{1,4}$, Pieter-Jan Wyndaele (D) ${ }^{1,5}$, Markus Hartmut Heyne (iD) ${ }^{1,2,5}$, Jianran Zhang ${ }^{1}$, Patrick C. With ${ }^{1}{ }^{1,6}$, Sreetama Banerjee ${ }^{1}$, Erik C. Neyts (iD $^{2}$, Inge Asselberghs ${ }^{1}$, Dennis $\operatorname{Lin}^{1}$ and Stefan De Gendt ${ }^{1,5}$

The cleaning of two-dimensional (2D) materials is an essential step in the fabrication of future devices, leveraging their unique physical, optical, and chemical properties. Part of these emerging 2D materials are transition metal dichalcogenides (TMDs). So far there is limited understanding of the cleaning of "monolayer" TMD materials. In this study, we report on the use of downstream $\mathrm{H}_{2}$ plasma to clean the surface of monolayer $\mathrm{WS}_{2}$ grown by MOCVD. We demonstrate that high-temperature processing is essential, allowing to maximize the removal rate of polymers and to mitigate damage caused to the $\mathrm{WS}_{2}$ in the form of sulfur vacancies. We show that low temperature in situ carbonyl sulfide (OCS) soak is an efficient way to resulfurize the material, besides hightemperature $\mathrm{H}_{2} \mathrm{~S}$ annealing. The cleaning processes and mechanisms elucidated in this work are tested on back-gated field-effect transistors, confirming that transport properties of $\mathrm{WS}_{2}$ devices can be maintained by the combination of $\mathrm{H}_{2}$ plasma cleaning and OCS restoration. The low-damage plasma cleaning based on $\mathrm{H}_{2}$ and OCS is very reproducible, fast (completed in a few minutes) and uses a $300 \mathrm{~mm}$ industrial plasma etch system qualified for standard semiconductor pilot production. This process is, therefore, expected to enable the industrial scale-up of 2D-based devices, co-integrated with silicon technology.

npj 2D Materials and Applications (2021)5:17; https://doi.org/10.1038/s41699-020-00197-7

\section{INTRODUCTION}

Two-dimensional transition metal dichalcogenides (TMDs) such as $\mathrm{MoS}_{2}$ and $\mathrm{WS}_{2}$ have attracted significant interest due to their unique properties and the relevance to numerous applications, particularly as synergistic combination with silicon-based nanosystems ${ }^{1}$. Field-effect transistors with channels made of atomically thin 2D semiconductors are less prone to short channel effects due to superior gate control over the channel, which allows device scaling to the few-nanometer scale ${ }^{2}$. Recently, the specific properties of these materials were exploited to create dopingfree logic functions based on polarity-controllable 2D devices ${ }^{3}$. Owing to the absence of surface dangling bonds, TMDs can be assembled in heterostructures with sharp interfaces and specific band alignment properties. Low power tunnel field-effect transistors are among the potential applications of such Van der Waals heterostructures ${ }^{2,4}$. While exceptional mechanical, optical, and electronic properties of TMDs have been demonstrated on mechanically exfoliated flakes, large-scale integration of these materials into sophisticated devices remains challenging. The wafer-scale fabrication process involves multiple technological steps such as deposition, surface cleaning, doping, and etching. Owing to the extreme sensitivity and fragility of $2 \mathrm{D}$ crystals in comparison to classical bulk systems, all processing technologies have to be reexamined. Significant progress has been made in the field of large-area synthesis of TMD layers. Most common deposition techniques include sulfurization ${ }^{4-6}$, atomic layer deposition $^{7,8}$, chemical vapor deposition with solid precursors ${ }^{9}$, and metal-organic precursors ${ }^{10}$. Among the above-mentioned approaches, metal-organic chemical vapor deposition (MOCVD) provides the best results in terms of grain size, thickness control and film uniformity. It is important to note that the deposition of high-quality TMD films involves high growth temperatures that are not compatible with the back-end of line (BEOL) processing requirements (temperature budget typically limited to $400^{\circ} \mathrm{C}$ ). Moreover, specific growth templates such as sapphire are needed to reach epitaxial film quality. Thus, a transfer from the growth substrate to the target substrate is required for subsequent integration. Surface contamination of 2D layers by polymer residues (e.g., PMMA) during the transfer has been a critical problem since the emergence of large-area CVD graphene ${ }^{11,12}$. Resist masks used for patterning and atmospheric contamination are other sources of hydrocarbon residues on 2D materials. In atomically thin layers, surface effects are dominant and surface contaminants, even at the lowest level, uncontrollably modify the properties of $2 \mathrm{D}$ crystals via charge transfer, strain and doping effects. Therefore, surface cleaning is a critical step in integration of synthetic 2D materials. Conventional wet cleaning, using acetone or other solvents, is not able to fully remove polymer residues $^{11,13,14}$. Moreover, wet processing of large-area 2D films may result in delamination or unintentional doping. From the manufacturing perspective, wet cleaning has a very low throughput and it generates a lot of solvent waste (typically an acetone soak of a few hours is used). Alternatively, thermal annealing of 2D materials leads to severe material modification at temperatures exceeding $200{ }^{\circ} \mathrm{C}^{12,15}$, which limits its applicability (sulfur loss for TMDs, heavy hole doping for Graphene). The lack of an efficient technique for low-damage cleaning of TMDs is, therefore, a major roadblock for their successful integration. It has been shown

\footnotetext{
${ }^{1}$ Imec v.z.w., Kapeldreef 75, 3001 Leuven, Belgium. ${ }^{2}$ Department of Chemistry, University of Antwerp, Universiteitsplein 1, 2610 Antwerp, Belgium. ${ }^{3} 1$ Skobeltsyn Institute of Nuclear Physics, Moscow State University, GSP-1 Leninskie Gori 1, Moscow 119234, Russia. ${ }^{4}$ Department of Physics and Astronomy, KU Leuven, Celestijnenlaan $200 \mathrm{~F}$, 3001 Leuven, Belgium. ${ }^{5}$ Department of Chemistry, KU Leuven, Celestijnenlaan 200F, 3001 Leuven, Belgium. ${ }^{6}$ Leibniz Institute of Surface Engineering (IOM), Permoserstrasse 15, 04318 Leipzig, Germany. ${ }^{凶}$ email: marneffe@imec.be
} 
recently, that high density hydrogen plasmas are effective for cleaning polymer residues from single layer graphene ${ }^{13}$. Nevertheless, our recent experiments indicate that direct $\mathrm{H}_{2}$ plasma exposure is too aggressive towards TMDs. Indeed, ion bombardment, in combination with active plasma species, lead to rapid sulfur loss from the outmost S-layer, as evidenced for $\mathrm{MoS}_{2}{ }^{16}$.

In the present paper, we investigate the use of a downstream hydrogen plasma for selective removal of PMMA (as a model contaminant) from single layer MOCVD WS $\mathrm{W}_{2}$ films. PMMA is used routinely as a flexible adhesive film for the transfer of $2 \mathrm{D}$ materials from one substrate to another; but multiple other polymers are being considered for transfer ${ }^{17}$. In downstream plasma configuration, only long-lived species, namely hydrogen atoms, are allowed to interact with the treated surface. Reactive $\mathrm{H}$ atoms are efficient for chemical etching of various polymers ${ }^{13,18,19}$. However, atomic hydrogen is also known to be reactive towards TMDs. Sulfur vacancy formation upon $\mathrm{H}$ exposure of $\mathrm{MoS}_{2}$ and $\mathrm{WS}_{2}$ via $\mathrm{H}_{2} \mathrm{~S}$ release has been reported in experiments ${ }^{20,21}$ and predicted in simulations ${ }^{22}$. The main challenge for dry cleaning of $\mathrm{WS}_{2}$ is to avoid sulfur loss while etching polymer residues. In this work, we demonstrate that the substrate temperature is a critical parameter during the cleaning process, since it governs both polymer removal and $\mathrm{WS}_{2}$ damage by reactive $\mathrm{H}$ atoms. Lost sulfur can be restored by soaking the S-depleted surface under low-pressure carbonyl sulfide (OCS) atmosphere.

\section{RESULTS AND DISCUSSION}

\section{Irreversibly adsorbed PMMA residue layer at the interface}

The first part of this study investigates the nature of PMMA residues and their removal using plasma-based methods. Polymer residues on $2 \mathrm{D}$ materials appear mainly after transfer from the growth substrate to the target substrate, using a rigid carrier wafer or flexible support layer, and one or multiple adhesive layers. After spin-coating the adhesive layer on the $2 \mathrm{D}$ layer and applying the rest of the transfer stack, the $2 \mathrm{D}$ layer is delaminated from the growth substrate, the entire stack is transferred to the target wafer and the transfer stack is removed. In the last step, the adhesive layer is removed from the $2 \mathrm{D}$ layer by a wet clean. This process has recently been upscaled to $300 \mathrm{~mm}$ wafers ${ }^{23}$. From this complex sequence, the adhesive application and removal is the most critical, which can be simulated by a three-stage experiment: first PMMA spin-coating on the 2D layer, then solvent bake-out, and finally PMMA removal using a solvent clean. We use PMMA as adhesive layer in contact with the 2D material, because it is easy to spin-cast, has good adherence, is continuous, non-damaging to the $2 \mathrm{D}$ materials and can be dissolved in acetone. The processing details are described in the experimental section of this paper (see methods). This simplified sequence resembles very much the procedure used to create Guiselin brushes ${ }^{24}$. Guiselin brushes appear at the interfaces between a non-repulsive wall and a semidilute polymer solution, and correspond to irreversibly adsorbed polymer layers. At a given temperature, each individual monomer can thermally detach from the surface; however, at chain level, due to connectivity, the number of simultaneously connected monomers remains too high for the polymer to detach from the surface, during timescales of interests. The kinetics of adsorption depends on the experimental parameters (temperature, solvent, polymer nature, polymer mass density, etc.) and leads to a layering of the polymer film at the interface, with varying chain conformation, connectivity to the wall, density-see the extensive review ${ }^{25}$ for more insight into this phenomenon. Of particular interest is the density increase at the interface ${ }^{26}$. In order to better understand the nature of the residual PMMA films that might appear during the processing of 2D films, an experiment has been defined, simulating the process conditions occurring during 2D transfer. A PMMA sol (anisole) was spin-casted on a Si substrate, afterwards annealed for various times and temperatures (above $T_{\mathrm{g}}$ ), and then cleaned with acetone. The thickness of the resulting PMMA layer versus the annealing time is shown in Fig. 1(a), for two different annealing temperatures, $135^{\circ} \mathrm{C}$ and $160^{\circ} \mathrm{C}$. During $2 \mathrm{D}$ transfer with wet adhesive removal, the PMMA is standardly baked for $5 \mathrm{~min}$ at $135^{\circ} \mathrm{C}$. The Fig. 1 (a) demonstrates that, even for short bake times, the minimal measured PMMA thickness is around $\sim 0.7-0.8 \mathrm{~nm}$, irrespective of the used temperature. Depending on the temperature, the thickness grow first following a linear law (full lines), then becomes logarithmic (dashed lines), eventually reaching a saturation "equilibrium" thickness $\Gamma^{\infty}$ (reached after $\sim 7.5 \mathrm{~h}$ at $160^{\circ} \mathrm{C}$ ). This fully corresponds to the description available in literature 27,28 : first, a linear regime with a large number of free sites available on the silicon surface for the attachment of new PMMA chains. It is followed by a second regime where the number of attachment sites decrease. At this stage, chains attachment becomes more sparse with long average polymer loops separating anchored monomers, less favorable from an energetic perspective, leading to a continuous reduction of the adsorption rate. Finally, this regime saturates to the equilibrium adsorbed amount $\Gamma^{\infty}$. Essentially, the Fig. 1 (a) indicates that an irreversibly adsorbed PMMA layer will always form on the surface, with thickness ranging from 0.8 to $3.5 \mathrm{~nm}$, depending on the conditions of spin-coating and subsequent thermal treatment. This layer, in the immediate vicinity of the interface, has a higher density than in the bulk polymer, and is therefore difficult to remove using classical solvents. This interface effect has been quantified in other systems, giving a density increase of up to $5-7 \%$ over a boundary layer of $\sim 12 \mathrm{~nm}^{29}$ for Teflon on silicon nitride.

Plasma-based polymer removal techniques have been explored for a few decades, with a primary focus on photoresist strip, selective to Silicon- or Germanium-based substrates. The requirements of fast removal rate and isotropy of the process, have led technologists to favor the use of downstream plasma systems equipped with high-temperature wafer holder (up to $350^{\circ} \mathrm{C}$ ) and cooled chamber walls. For TMD cleaning, $\mathrm{H}_{2}$-based downstream plasma system (DPS) processes offer the benefit of being nonoxidizing, which is required to avoid immediate desulfurization by the formation of volatile $\mathrm{SO}_{2}$ and subsequent tungsten oxidation. The major drawback of $\mathrm{H}_{2}$-based DPS is their low resist removal rate relative to $\mathrm{O}_{2}$-based discharges.

The Fig. 1(b, c) aim at better understanding the removal mechanism of the PMMA, when exposed to a downstream $\mathrm{H}_{2}$ plasma. The evolution of PMMA thickness as a function of the DPS etch time is shown in Fig. 1(b), for three substrate temperatures. The use of scaled coordinates allows to circumvent the large difference in absolute etch rates, and highlight different etch regimes. An overlay is achieved for all three curves at $d / d_{\max } \gtrsim$ 0.01 , i.e., $d \gtrsim 6-10 \mathrm{~nm}$, indicating that the polymer etching, although being different in rate, follows the same linear removal mechanism, i.e., without formation of a top carbon-rich crust slowing down the reaction rate. For a thickness below $\sim 6 \mathrm{~nm}, \mathrm{a}$ discrepancy appears, where the polymer removal is slower, especially at higher temperatures, $170-250^{\circ} \mathrm{C}$. Another insight is given by Fig. 1(c), showing an Arrhenius plot of the mean removal rate versus temperature. It is found that in the chosen plasma conditions the etch rate of PMMA increases over more than three orders of magnitude, from $\sim 0.06$ to $\sim 100 \mathrm{~nm} / \mathrm{s}$ when the substrate temperature is increased from 60 to $300^{\circ} \mathrm{C}$. This graph allows to discriminate two etch regimes: for $T<120^{\circ} \mathrm{C}$, an activation energy of $E_{\mathrm{a}}=0.64 \mathrm{eV}$ can be calculated, while for $T>120^{\circ} \mathrm{C}$, another etch regime appears with lower apparent activation energy.

PMMA is a low-density polymer showing free volume and/or intrinsic microporosity, which, due to these characteristics, has been recently used as a template for vapor phase infiltration synthesis $^{30,31}$. Polymer microporosity can impact the material removal rate during the plasma etch process, by enhancing the 

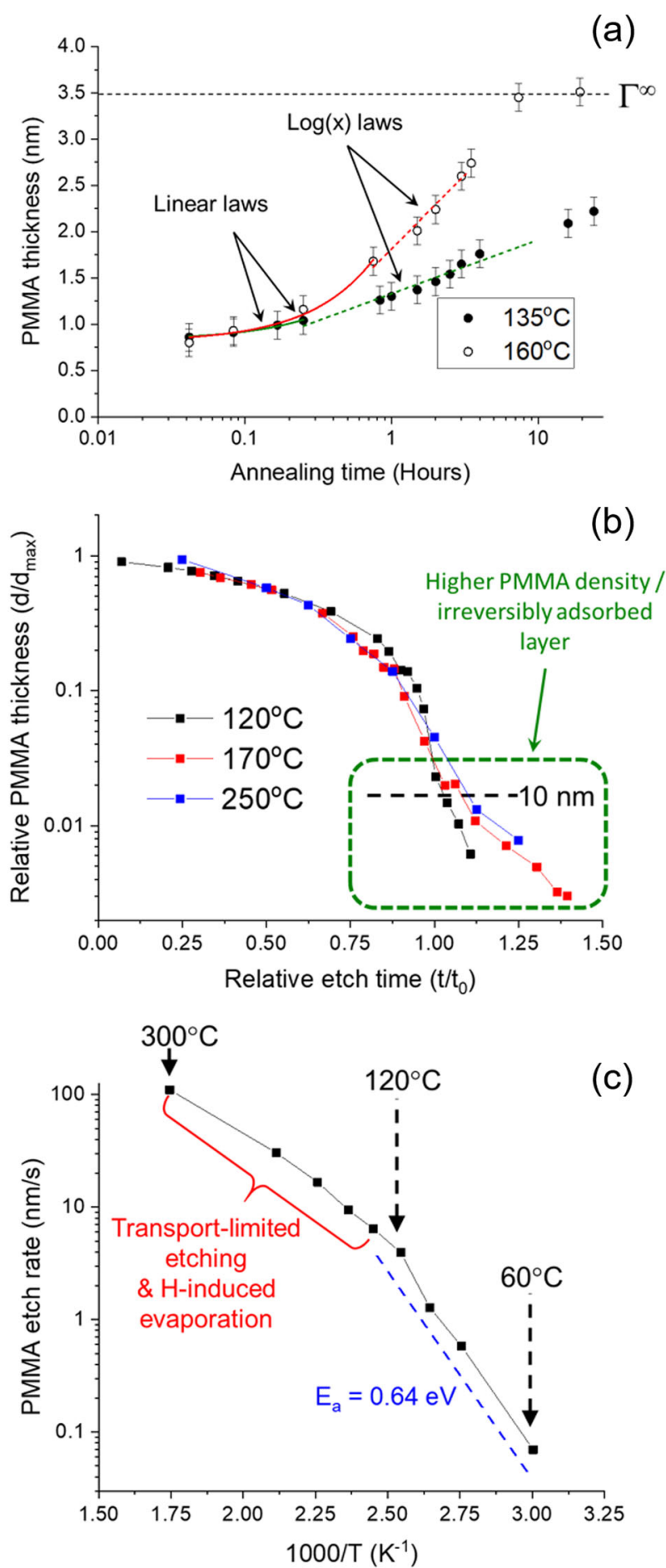

Fig. 1 Removal characteristics of the PMMA film on Si substrates. a After overnight clean in acetone, adsorption kinetics for monolayer PMMA films, indicating the presence of irreversibly adsorbed layer, not dissolved by the solvent. $\mathbf{b}$ Scaled thickness-time evolution of PMMA thick films exposed to $\mathrm{H}_{2}$ downstream plasma, at different substrate temperatures; $d_{\max } \sim 570 \mathrm{~nm}$ and $t_{0}=t(d=$ $0.03 \times d_{\text {max }}$ ). Below $\sim 10 \mathrm{~nm}$, the presence of the irreversibly adsorbed layer causes a change in etch mechanism. c Arrhenius plots of the average etch rate in $\mathrm{H}_{2}$ downstream plasma, obtained by linear fitting of the curve shown in $\mathbf{b}$, for $d>50 \mathrm{~nm}$. A threshold is visible around $T \sim 120^{\circ} \mathrm{C}$. The calculated activation energy is indicated on the graph. Error bars represent confidence intervals $(\mathrm{Cl})$. sub-surface mobility of reactive plasma radicals. Such phenomenon might lead to 3D etching, i.e., chemical reactions are not limited to the very top surface but also occur over a certain thickness into the bulk, i.e., increasing the removal rate compared to a pure 2D etch mechanism. This 3D etching stops when the etch front reaches the irreversibly adsorbed layer, which has a higher density, reduced free volume and microporosity. From this interface, the sub-surface mobility of $\mathrm{H}^{*}$ radicals gets reduced, leading to a sudden decrease in removal rate, as observed in the Fig. 1(b). This becomes particularly visible as the system is heated far above $T_{\mathrm{g}}$.

The etch reactions of PMMA under a flux of $\mathrm{H}$ atoms have been studied in-depth by using mass spectroscopy ${ }^{19}$. The major decomposition products of a PMMA film at $147^{\circ} \mathrm{C}$ were highlighted as being essentially methane and $\mathrm{CO}$, therefore emphasizing the following reaction process

$$
\mathrm{C}_{5} \mathrm{H}_{8} \mathrm{O}_{2}+4 \mathrm{H} \rightarrow 2 \mathrm{CO}+3 \mathrm{CH}_{4}
$$

In the DPS configuration used in this work (see "methods"), the maximum flux of $\mathrm{H}$ atoms in the plasma chamber is $3.4 \times 10^{18}$ atoms/s (considering $100 \% \mathrm{H}_{2}$ dissociation, not likely in such a system; $1 \mathrm{sccm} \mathrm{H} \mathrm{H}_{2}=7.5 \times 10^{7} \mathrm{~mol} / \mathrm{s}$ ). The rate of carbon release in the chamber can be easily estimated, taking into account a PMMA $\left(\mathrm{C}_{5} \mathrm{H}_{8} \mathrm{O}_{2}\right)$ density of $1.18 \mathrm{~g} / \mathrm{cm}^{3}$, as well as the exposed surface area (small piece of Si coated with PMMA and placed on a $300 \mathrm{~mm}$ carrier Si wafer, or full $300 \mathrm{~mm}$ wafer coated with PMMA) and measured etch rate (as shown in Fig. 1(c)). Depending on the exposed PMMA area and processing temperature, the carbon removal will vary between $10^{15}$ atoms $/ \mathrm{s}\left(1 \mathrm{~nm} / \mathrm{s}, 1 \times 1 \mathrm{~cm}^{2}\right.$ coupon) and $10^{20}$ atoms $/ \mathrm{s}$ ( $100 \mathrm{~nm} / \mathrm{s}$, full wafer). Comparing with the maximum flux of $\mathrm{H}$ atoms of the order of $10^{18}$ atoms $/ \mathrm{s}$ calculated above, it is obvious that, at the highest etch rate, i.e., high temperature, the system is getting into a reactant-poor reaction, or transport limited mode. In this situation, the concentration of $\mathrm{H}$ radicals at the surface drops, because $\mathrm{H}$ atoms are consumed rapidly at the surface due to the fast etching. At low temperature, $T<120^{\circ} \mathrm{C}$, the system remains in a reaction-limited mode, causing the apparently high activation energy observed in Fig. 1(c). At high temperature, there is an imbalance between the calculated $\mathrm{H}$ inbound flux and $\mathrm{C}$ outbound flux, which seems like an inconsistency if we restrict our analysis to equation (1). However, it is well-known that, in vacuum, PMMA starts to decompose and desorb from $\sim 250^{\circ} \mathrm{C}$ onwards (thermogravimetric data). Therefore, it can be assumed that in the present case, at high-temperature, PMMA is removed by hydrogen-induced volatilization, i.e., becomes fragmented by the (low) flux of reactive $\mathrm{H}$ atoms, leading to an enhanced desorption of molecular volatile fragments.

\section{Interaction of $\mathbf{H}$ atoms with pristine $\mathbf{W S}_{2}$, and OCS healing}

The effect of the downstream hydrogen plasma exposure on pristine as-grown $\mathrm{WS}_{2}$, without PMMA coating, is investigated here to assess and understand the $\mathrm{WS}_{2}$ damage mechanism. Atomic force microscopy (AFM) topography images and reference photoluminescence $(\mathrm{PL})$ measurements of pristine monolayer $\mathrm{WS}_{2}$ are shown in the paragraph describing the methods. Photoluminescence spectra acquired after a $6 \mathrm{~s}$ remote $\mathrm{H}_{2}$ plasma strip performed at different substrate temperatures reveal that the $\mathrm{PL}$ signal is quenched, especially when the treatment temperature is decreased from 300 to $120^{\circ} \mathrm{C}$ (Fig. 2(a)). Photoluminescence properties are related to the crystalline quality of $\mathrm{WS}_{2}$ and the reduction of the $\mathrm{PL}$ intensity is an evidence of defect generation. Quenching of photoluminescence associated with the presence of sulfur vacancies in monolayer $\mathrm{WS}_{2}$ has been reported in ref. ${ }^{32}$ showing that PL intensity is inversely proportional to the density of defects. Red shift of PL spectra (by $\sim 10 \mathrm{meV}$ ) observed at 

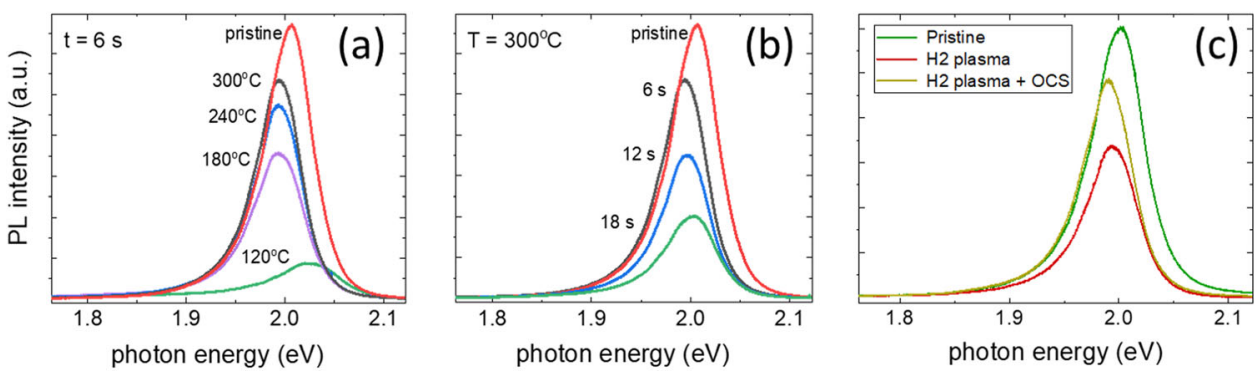

Fig. 2 Impact of DPS $\mathbf{H}_{2}$ treatments on as-grown $\mathbf{W S}_{2}$ on $\mathrm{Si}_{\mathbf{S}} \mathrm{SiO}_{2}$. a PL emission spectra of $\mathrm{WS}_{2}$ after a $6 \mathrm{~s}$ downstream plasma strip performed at different substrate temperatures; $\mathbf{b} \mathrm{PL}$ emission spectra of $\mathrm{WS}_{2}$ exposed to the downstream plasma at $300{ }^{\circ} \mathrm{C}$ during 6,12 , and $18 \mathrm{~s}$; c combined effects of DPS $\mathrm{H}_{2}\left(12 \mathrm{~s}\right.$ at $\left.300^{\circ} \mathrm{C}\right)$ followed by OCS soak $\left(240 \mathrm{~s}\right.$ at $\left.60^{\circ} \mathrm{C}\right)$.

treatment temperatures between 180 and $300^{\circ} \mathrm{C}$ is ascribed to the presence of sulfur vacancies as discussed in ref. ${ }^{33}$.

The results shown in Fig. 2(a) indicate that the downstream $\mathrm{H}_{2}$ plasma treatment at elevated surface temperatures creates fewer sulfur vacancies. In this study, the flux of $\mathrm{H}$ atoms towards the surface is fixed and does not depend on the temperature of the substrate. Therefore, the probability of sulfur vacancy generation in reactions between $\mathrm{WS}_{2}$ and $\mathrm{H}$ decreases with temperature. In other words, the rate of $\mathrm{WS}_{2}$ damage by atomic hydrogen demonstrates negative temperature dependence. This is the opposite of what one would expect for a thermally activated chemical process, like for instance polymer etching, see Fig. 1(c)).

This unexpected temperature dependence is qualitatively explained by density functional theory (DFT) calculations of $\mathrm{WS}_{2}$ surface chemistry, see Supplementary Discussion and Supplementary Fig. 1 for more details. When a WS 2 surface is exposed to a $\mathrm{H}$ flux, the formation of $\mathrm{H}_{2} \mathrm{~S}$ is the main route for sulfur depletion. $\mathrm{H}$ is predicted to adsorb preferentially on surface $S$ atoms, forming a surface $\mathrm{SH}_{\text {surf }}$ group with a calculated $\mathrm{H}$ adsorption energy $E_{\mathrm{H}}$ in the range of $0.4 \mathrm{eV}$ (DFT/AO) to $0.53 \mathrm{eV}$ (DFT + U/PW). Moreover, the clustering of two adsorbed $\mathrm{H}$ atoms, resulting in a $\mathrm{H}_{2} \mathrm{~S}_{\text {surf }}$ complex is energetically favored over two isolated $\mathrm{SH}_{\text {surf }}$ groups, opening the way to the desorption of a $\mathrm{H}_{2} \mathrm{~S}$ molecule (also energetically favored), leaving a sulfur vacancy behind. During the reaction of an incident $\mathrm{H}$ atom with $\mathrm{SH}_{\text {surf, the energy barriers of }}$ key processes (see Supplementary Discussion) such as $\mathrm{H}$ migration on the surface, clustering, $\mathrm{H}_{2} \mathrm{~S}$ desorption and $\mathrm{H}_{2} \mathrm{~S}(\mathrm{~g})$ release are low, indicating that even moderate surface $\mathrm{H}$ coverage can cause sulfur depletion at low temperature. In order to suppress the formation of sulfur vacancies, the $\mathrm{H}$ coverage must therefore remain sufficiently low. This can be achieved by increasing the temperature of the surface. Assuming that the equilibrium $\mathrm{H}$ coverage is proportional to $\exp \left(-E_{\mathrm{H}} / k_{\mathrm{B}} T\right)$, with $k_{\mathrm{B}}$ the Boltzmann constant, higher temperatures will counteract the (exothermic) $\mathrm{H}$ adsorption process. With a $\mathrm{H}$ adsorption energy of $0.53 \mathrm{eV}$ (DFT + $\mathrm{U} / \mathrm{PW}$ model), increasing the temperature from 393 to $573 \mathrm{~K}$ (120 to $300^{\circ} \mathrm{C}$ ) leads to a decrease of $\mathrm{H}$ coverage by two orders of magnitude. Similarly, following Arrhenius' law, the H desorption rate will increase by the same extent. The probability of $\mathrm{H}$ clustering, which requires the stoichiometric combination of two surface $\mathrm{H}$, is proportional to the square of the $\mathrm{H}$ coverage, and is hence strongly suppressed upon increase of the temperature. Such a dramatic decrease of the $\mathrm{SH}_{\text {surf }}$ coverage (and hence the rate of $\mathrm{H}_{2} \mathrm{~S}_{\text {surf }}$ formation and $\mathrm{H}_{2} \mathrm{~S}(\mathrm{~g})$ release) explains the unexpected reduction of the $\mathrm{WS}_{2}$ damage at elevated surface temperatures observed in the experiments. Besides the discussed processes of $\mathrm{H}$ adsorption and $\mathrm{H}_{2} \mathrm{~S}$ formation, several other mechanisms can result in the removal of adsorbed $\mathrm{H}$, and hence help to prevent defect formation. Such mechanisms include Langmuir-Hinshelwood recombination of two surface $\mathrm{H}$ atoms, and Eley-Rideal recombination of $\mathrm{H}(\mathrm{g})$ and $\mathrm{SH}_{\text {surf, }}$, both leading to the formation and release of $\mathrm{H}_{2}(\mathrm{~g})$. Such mechanisms will also be accelerated at high temperature, and contribute to a reduction of the $\mathrm{SH}_{\text {surf }}$ coverage. Additional DFT simulations (not presented here) showed that $\mathrm{H}$ reactivity on $\mathrm{MoS}_{2}$ monolayers is similar to the case of $\mathrm{H}-\mathrm{WS}_{2}$ system. It is therefore expected that remote hydrogen plasma will also be efficient for cleaning of $\mathrm{MoS}_{2}$ and other TMDs.

From the $\mathrm{WS}_{2}$ cleaning perspective, elevated treatment temperatures ensure higher PMMA removal efficiency, as well as better selectivity towards $\mathrm{WS}_{2}$. Figure 2(b) demonstrates that extended plasma exposure at $300^{\circ} \mathrm{C}$ leads to a steady decrease of the PL intensity as a result of defect generation. However, even after $18 \mathrm{~s}$ of plasma treatment the overall shape of the PL spectrum remains unaffected, which means that the material retains its structural properties. Remote hydrogen plasma can, therefore, be employed not only for cleaning but also for a soft sulfur vacancy generation in TMD layers. Controllable production of sulfur vacancies is a promising route for doping, chemical activation, or contact engineering in TMDs.

Keeping the substrate temperature sufficiently high is essential to have efficient and selective polymer removal. Etch selectivity can be further improved by tuning the flux of $\mathrm{H}$ atoms. The rate of sulfur vacancy generation via $\mathrm{H}_{2} \mathrm{~S}$ release is proportional to the square of the incoming atomic hydrogen flux while PMMA decomposition is a first order process and its rate is a linear function of the $\mathrm{H}$ flux ${ }^{19}$. Hence, the reduction of hydrogen flux is expected to further improve etch selectivity towards $\mathrm{WS}_{2}$, while minimizing the $S$ vacancy formation.

Figure $2(a, b)$ show that high temperature and short treatment time can reduce the damage to the $\mathrm{WS}_{2}$ monolayer, however, damage cannot be fully suppressed, as a minimal exposure time is needed to ensure good cleaning. Using the $300{ }^{\circ} \mathrm{C}$ process studied in Figs. $1(\mathrm{~b}-\mathrm{c})$ and 2(a), a practical example can be estimated for a PMMA transfer layer of $500 \mathrm{~nm}$. For a removal rate of $100 \mathrm{~nm} / \mathrm{s}$, taking into account process uniformity of $\sim 50 \%$, the calculated total $\mathrm{H}_{2}$ plasma exposure time would be 8-10 s, i.e., some parts of the $\mathrm{WS}_{2}$ surface would be exposed to the $\mathrm{H}_{2}$ plasma during $3-5 \mathrm{~s}$, causing a non-negligible sulfur loss. Once a sulfur vacancy is created, ambient exposure leads to metal oxidation, $\mathrm{WO}_{x}$, which is difficult to reduce. In order to compensate for the sulfur loss, resulfurization is therefore necessary. Annealing in $\mathrm{H}_{2} \mathrm{~S}$ has been used for the synthesis of metal disulfide layers ${ }^{4-6}$. The reduction of metal-oxide $\left(\mathrm{MoO}_{3}\right)$ typically requires temperatures in excess of $800^{\circ} \mathrm{C}$ to enable full and efficient conversion to a sulfide ${ }^{34}$. Also, $\mathrm{H}_{2} \mathrm{~S}$ plasma treatment was tried with contradictory results ${ }^{35,36}$. To avoid ambient exposure, it is interesting to perform resulfurization directly after $\mathrm{H}_{2}$ exposure, without air break, ideally in situ. Multiple sources of sulfur could be considered, with a preference to halogen-free molecules so as to avoid etching the W layer; an interesting candidate is carbonyl sulfide $(\mathrm{O}=\mathrm{C}=\mathrm{S})$, which is less toxic than $\mathrm{H}_{2} \mathrm{~S}$ and adopted by the plasma etch community for its use as sidewall passivant ${ }^{37}$. The injection of OCS in a plasma would lead to dissociation of the molecule, and release oxidizing components that could be harmful to the exposed $\mathrm{WS}_{2}$. On the other hand, a flux of molecular OCS could enable surface reactions 

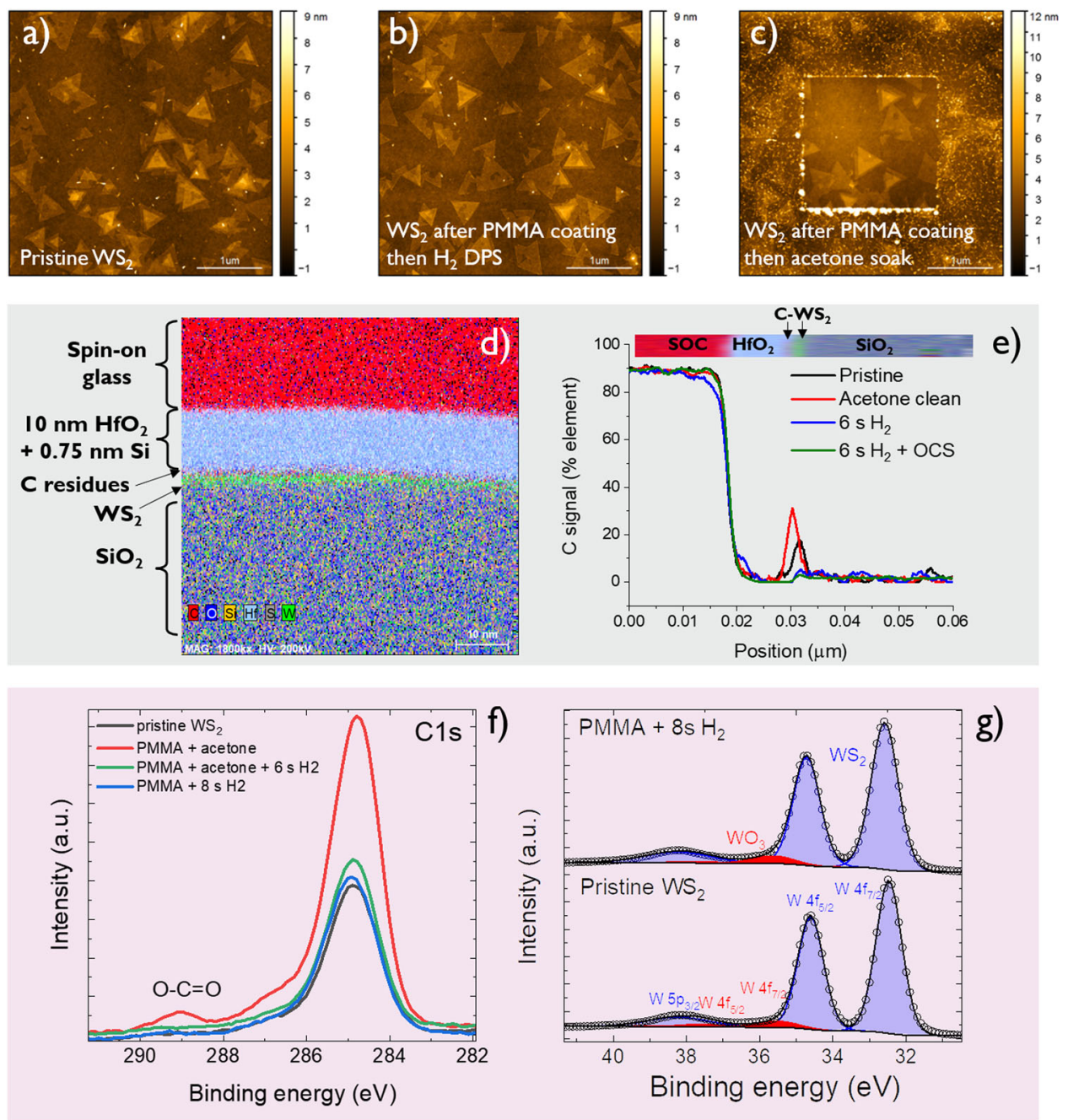

Fig. 3 Morphology and surface chemistry of $\mathbf{W S}_{\mathbf{2}}$ films before and after cleaning treatments. Topography AFM images: a pristine as-grown material; b $200 \mathrm{~nm}$ PMMA removed by $8 \mathrm{~s} \mathrm{H}$ plasma strip at $300^{\circ} \mathrm{C}$; c PMMA removed by $2 \mathrm{~h}$ acetone soak, A $2 \mu \mathrm{m} \times 2 \mu \mathrm{m}$ square in the center of the image was mechanically cleaned by the AFM tip. Cross-section TEM imaging: d illustrative EDS-TEM cross-section image of a WS ${ }_{2}$ sample after close-coupled deposition of a $10 \mathrm{~nm} \mathrm{HfO}$ layer (using a initiation layer of $0.75 \mathrm{~nm}$ Si deposited by PVD); e integrated carbon elemental profiling for various cleaning treatments. Chemical characterization using XPS: f C1s spectra measured after different PMMA removal approaches; $\mathbf{g}$ W4f core spectra of pristine $\mathrm{WS}_{2}$ and after plasma removal of $200 \mathrm{~nm}$ PMMA.

due to the high reactivity of dangling $W$-bonds at a sulfur vacancy and achieve healing. The Fig. 2(c) shows the impact of OCS healing, applied after $\mathrm{H}_{2}$ plasma treatment, without air break (in the same processing platform, but different chamber, after transfer in a low-pressure $\mathrm{N}_{2}$ atmosphere). It indicates that the $W_{2}$ damage caused by $\mathrm{H}_{2}$ dry cleaning can be partly healed by OCS. This is supported by water contact angle (WCA) measurements, that allow to determine the surface hydrophobicity of $\mathrm{WS}_{2}$ monolayer. After $35 \mathrm{~s}$ of exposure to a $\mathrm{H}_{2}$ DPS plasma cleaning, which is an extensive treatment as compared to the few seconds needed to remove PMMA thickness of few hundreds of nanometers, the $\mathrm{WS}_{2}{ }^{\prime}$ 's hydrophobicity shows a drop of $\sim 25^{\circ}$. This is indicative of an increase of surface polarity, caused by the formation of hydrophilic $\mathrm{WO}_{x}$ as evidenced by X-ray photoelectron spectroscopy (XPS). By combining the plasma dry clean with in situ OCS soaking, the WCA drop is $60 \%$ lower, indicative of a partially recovered low surface polarity.

The mechanism of OCS healing is clarified by DFT calculations, see Supplementary Discussion, Supplementary Fig. 3, and ref. ${ }^{38}$ for more details. While approaching the $\mathrm{WS}_{2}$ surface at a $\mathrm{S}$ vacancy location, and impinging OCS molecule can follow two reaction pathways. As a first option, it can dissociate into $\mathrm{CO}$ and
$\mathrm{S}$, filling the S-vacancy with release of volatile CO. A second option corresponds to the OCS reacting with $\mathrm{H}$ atoms adsorbed on the $\mathrm{WS}_{2}$ monolayer, forming an intermediate OCSH radical, which subsequently can dissociate on the $S$ vacancy with the elimination of a volatile CO molecule. DFT calculations indicate that the first pathway has an activation barrier of $\sim 1.1 \mathrm{eV}$, while the second option has a barrier of only of $0.2-0.3 \mathrm{eV}$, making it significantly more favorable. As will be shown in another paper (to be published), the OCS has additional benefit linked to the enhancement of charge injection through grain edges and boundaries.

\section{Selective removal of PMMA}

Figure 3(a-c) show AFM images of $\mathrm{WS}_{2}$ after PMMA removal by $\mathrm{H}_{2}$ plasma $\left(8 \mathrm{~s}\right.$ at $\left.300^{\circ} \mathrm{C}\right)$ and by a conventional acetone soak $(2 \mathrm{~h}$ at $20^{\circ} \mathrm{C}$ ), together with an image of as-grown material. Acetone cleaning is not able to fully remove PMMA, leaving residues that preferentially decorate grain edges and boundaries and reach a few nanometers in height. By contrast, the $\mathrm{H}_{2}$ plasma cleaning yields a clean surface, free from PMMA contamination and indistinguishable from the pristine material. A root-mean-square (RMS) surface roughness of $0.27 \mathrm{~nm}$ was measured on both 
pristine and plasma-cleaned samples. Moreover, no signs of $\mathrm{WS}_{2}$ etching or damage by atomic hydrogen exposure can be observed by AFM, which proves excellent process selectivity.

These results are confirmed by cross-section transmission electron microscopy (TEM) analysis on $\mathrm{WS}_{2}$ samples, which were spin-coated with PMMA, then exposed to three cleaning conditions; acetone wet clean ( $2 \mathrm{~h}$ dipping), $\mathrm{H}_{2}$ downstream plasma and $\mathrm{H}_{2}$ downstream plasma coupled to $240 \mathrm{~s}$ OCS soaking. An additional pristine $W_{2}$ reference sample did not get PMMA coating nor any clean before capping. All samples were capped with $10 \mathrm{~nm} \mathrm{HfO}$ layer deposited by atomic layer deposition (ALD), in order to better image the residual carbon layer present on the surface, and separate it from the spin-on carbon film deposited during the TEM preparation. The high-k nucleation was enabled by the deposition of $0.75 \mathrm{~nm}$ Si using physical vapor deposition (PVD), which is then oxidized by ambient exposure. Dry cleaning and Si deposition were close-coupled so as to minimize surface contamination. Figure $3(\mathrm{~d})$ shows the energy dispersive spectroscopy (EDS) overlay of different elements present in the sample that was cleaned using acetone dip. The carbon residues on top of the $\mathrm{WS}_{2}$ monolayer are clearly visible, showing that acetone did not successfully remove all PMMA. This is compared to $\mathrm{H}_{2}$ plasmabased cleaning conditions in Fig. 3(e), which is an integrated element mapping along a vertical profile of TEM images as shown in Fig. 3(d). The acetone-cleaned sample has the highest level of carbon residues, $>30 \%$. The pristine reference sample shows $\sim 20 \%$ carbon, which is presumably adsorbed hydrocarbons from the ambient atmosphere accumulated over several days. Samples that were exposed to $\mathrm{H}_{2}$ plasma and $\mathrm{H}_{2}$ with OCS soak do not show any detectable $C$ residues. In the case of the OCS soak, the data demonstrates that after reaction with the surface and sulfur vacancy healing, OCS does not leave any $\mathrm{C}$ contamination on the $\mathrm{WS}_{2}$.

The efficiency of the $\mathrm{H}_{2}$ plasma cleaning is confirmed by XPS surface analysis comparing three PMMA removal strategies-see Fig. $3(f-g)$. The first approach is an overnight cleaning using acetone. The second approach is a fully dry plasma cleaning, where the entire $200 \mathrm{~nm}$ PMMA film is etched in $8 \mathrm{~s} \mathrm{H}_{2}$ plasma at $300^{\circ} \mathrm{C}$. A third alternatively is the wet-dry combination, where PMMA is first removed by acetone overnight followed by a $6 \mathrm{~s}$ plasma strip. Based on the PMMA etch rate $\left(100 \mathrm{~nm} / \mathrm{s}\right.$ at $\left.300^{\circ} \mathrm{C}\right)$, we estimate that the samples of the second and third approaches received an identical plasma over-etch time of $\sim 6 \mathrm{~s}$. As expected for the overnight acetone dip, XPS measurements of the C1s core peak (Fig. $3(\mathrm{f})$ ) indicate the presence of $\mathrm{O}-\mathrm{C}=\mathrm{O}$ bonds, which are a signature of PMMA residues ${ }^{13}$. This confirms that even an extended acetone soak is not able to fully remove PMMA. Plasma cleaning in turn yields $\mathrm{WS}_{2}$ surfaces free from $\mathrm{PMMA}$ residues with only airborne carbon contamination detected by XPS. Analysis of the tungsten core level spectra in Fig. $3(\mathrm{~g})$ indicates that as-grown $\mathrm{WS}_{2}$ contains a small fraction of $\mathrm{WO}_{3}$, which is formed on initially present sulfur vacancies and on grain boundaries during the material exposure to the ambient environment ${ }^{7,8}$. One can note that the fraction of $\mathrm{WO}_{3}$ in the material remains unchanged after the plasma exposure, which confirms that the sulfur loss caused by the plasma cleaning (and subsequent $\mathrm{WO}_{x}$ formation) is too low to be visible by XPS.

In the previous sections, it was shown that very resilient residues, corresponding to irreversibly adsorbed polymer chains, can be formed at the $\mathrm{WS}_{2}$-PMMA interface. These residues appear on other 2D materials, as shown in references ${ }^{11-13}$. In the case of $\mathrm{WS}_{2}$, PMMA residues form $3 \mathrm{D}$ islands that tend to accumulate along grain boundaries, possibly due to a stronger chemical bonding of PMMA on more reactive grain edges. These residues are not removed by any organic solvent and cannot be fully eliminated by annealing ${ }^{13}$. The etch rate of this interfacial PMMA layer in hydrogen plasma is significantly lower than that of bulk PMMA. However, no quasi-2D residue layers could be observed by

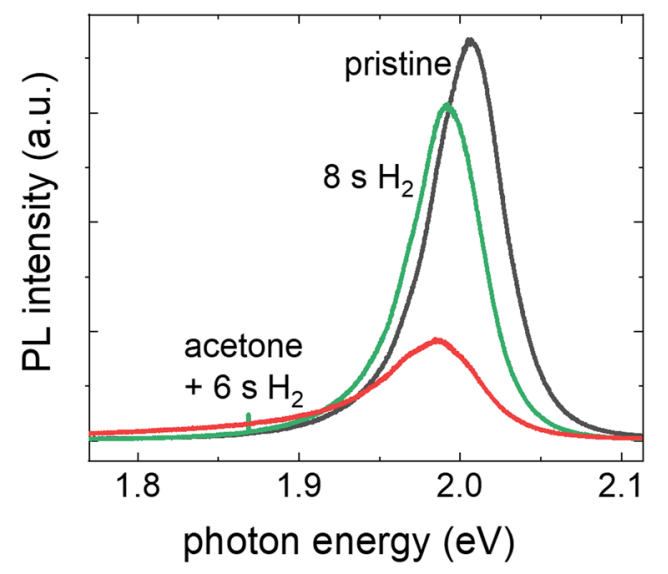

Fig. 4 After PMMA removal with a hydrogen plasma strip (8s $\mathrm{H}_{2}$ plasma), the PL spectra show only a minor change as compared with pristine $\mathbf{W S}_{2}$. Using an acetone soak followed by a $6 \mathrm{~s}$ plasma strip, the PL intensity drops significantly.

AFM, XPS and in atomic resolution EDS-TEM imaging (see Fig. 3(e)) and planar TEM. The data shown above prove that even strongly bonded PMMA residues can be efficiently removed from the $\mathrm{WS}_{2}$ surface by the downstream $\mathrm{H}_{2}$ plasma strip.

Photoluminescence (PL) measurements shown in Fig. 4 prove that dry removal of PMMA from $\mathrm{WS}_{2}$ does not generate more damage than just $\mathrm{H}_{2}$ plasma exposure of the pristine material (cf. Fig. 2). This indicates that PMMA etch by-products generated during the dry cleaning process are not attacking $\mathrm{WS}_{2}$. Surprisingly, the sample that was pre-cleaned with acetone prior to the $\mathrm{H}_{2}$ strip is more severely damaged. It shows a significant PL intensity drop with a low energy tail in the PL spectrum. Additional experiments showed that just an acetone soak of pristine $\mathrm{WS}_{2}$ (without PMMA) renders the material more fragile and prone to degradation under hydrogen plasma exposure. Such modification of $\mathrm{WS}_{2}$ reactivity can be attributed to a synergetic effect between hydrogen atoms and acetone residues on the $\mathrm{WS}_{2}$ surface or to acetone intercalation between $\mathrm{WS}_{2}$ and the growth substrate $\left(\mathrm{SiO}_{2}\right)$. Acetone cleaning of $2 \mathrm{D}$ materials is widely used and it is considered as soft and non-damaging. Nevertheless, the PL data shown in Fig. 4 demonstrate that acetone has a strong impact on the surface reactivity of $\mathrm{WS}_{2}$. From practical considerations, dry plasma cleaning of PMMA residues should not be combined with acetone soak. If applied alone, remote hydrogen plasma can fully eliminate PMMA with only a minor impact on the material properties.

\section{Effect of dry cleaning on $\mathbf{W S}_{\mathbf{2}}$ transport properties}

To assess the impact of the dry cleaning on the electrical properties of the $\mathrm{WS}_{2}$ film, back-gated field-effect transistors were fabricated, including PMMA cleaning by the proposed $\mathrm{H}_{2}$ downstream plasma, as well as resulfurization approaches. In order to assess the damage and restoration properties of the used processes, the devices were compared with reference sets, which did not get any PMMA coating nor removal. The goal of this exercise is to reproduce the reference electrical characteristics as closely as possible.

After PMMA spin-coating then hydrogen plasma clean $(8 \mathrm{~s}$ at $300^{\circ} \mathrm{C}$ ), two sulfur restoration routes are studied: the first one using ex situ $\mathrm{H}_{2} \mathrm{~S}$ annealing at various temperatures, and the second using in situ OCS soak. Owing to bandwidth in device fabrication and tool access, the experiment was performed using two sets of CVD WS $\mathrm{WS}_{2}$ samples, having slightly different pristine characteristics. The device fabrication is detailed in the experimental section of the paper (see "Methods"). Devices with channel length of $0.5-15 \mu \mathrm{m}$ and channel width of $104 \mu \mathrm{m}$ were fabricated 

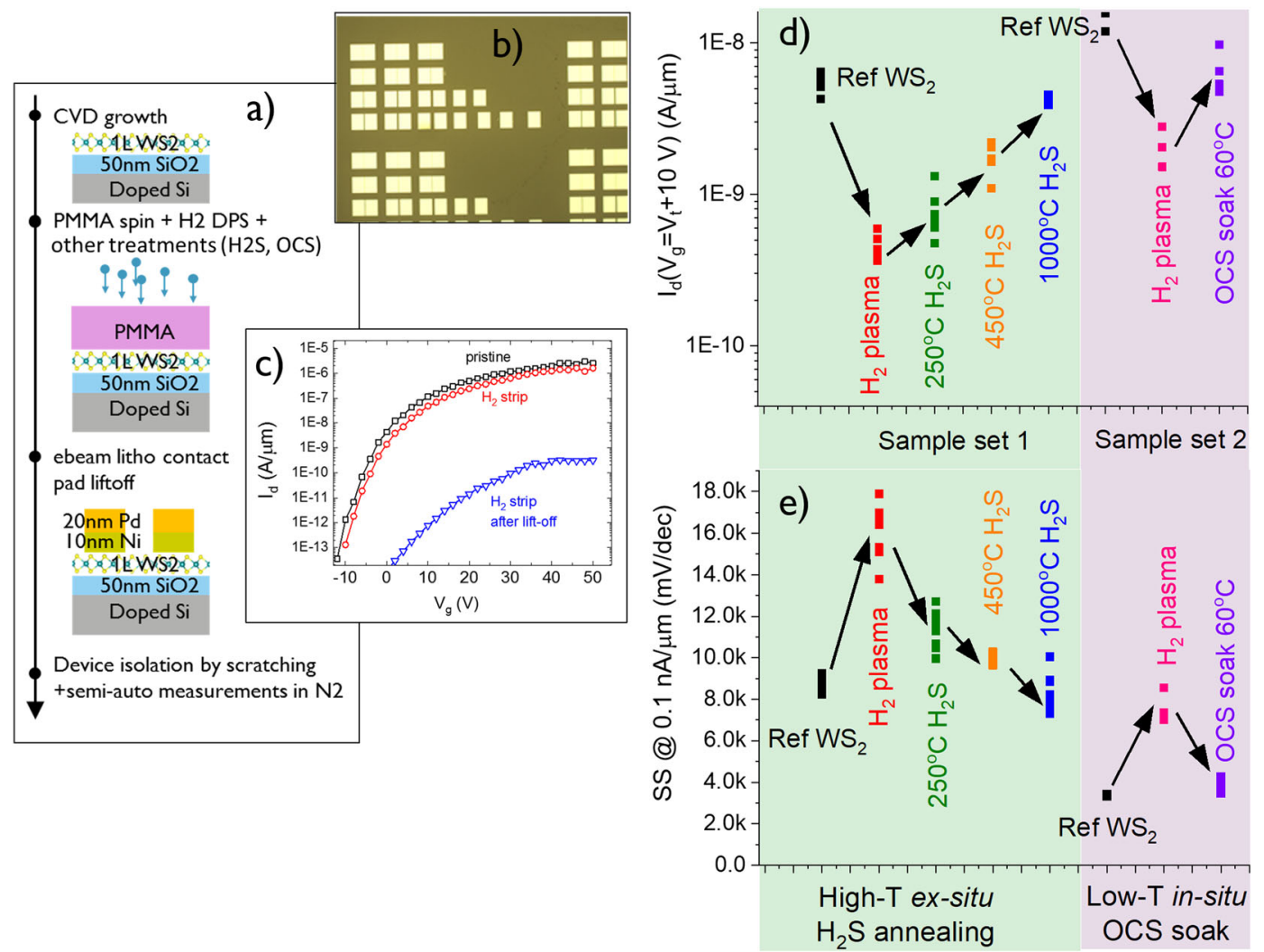

Fig. 5 Electrical characteristics of simple $\mathbf{W S}_{\mathbf{2}}$ devices. a Process flow used for fabrication of the devices; $\mathbf{b}$ Optical microscope image of fabricated devices after contact lift-off; $c l_{\mathrm{d}}-V_{\mathrm{g}}$ characteristics of back-gated devices with $500 \mathrm{~nm}$ channel length. $\mathrm{H}_{2}$ plasma exposure was performed either before device fabrication or after contact lift-off; $\mathbf{d}$ Drive current $I_{\mathrm{on}}=I_{\mathrm{d}}\left(V_{\mathrm{t}}+10 \mathrm{~V}\right)$ measured for all samples (15 $\mu \mathrm{m}$ channel length), as a function of annealing and soak conditions; e Sub-threshold swing SS (taken at $100 \mathrm{pA} / \mu \mathrm{m}$ ) measured for all samples, as a function of annealing and soak conditions. The experiment was conducted over two batches of $\mathrm{WS}_{2}$.

by electron beam lithography with PMMA resist, evaporation of $\mathrm{Ni} / \mathrm{Pd}$ contacts and lift-off in acetone. A schematic overview of the process flow is shown in Fig. 5(a) and optical microscope images of the resulting $\mathrm{WS}_{2}$ devices are shown in Fig. 5(b). Note that the finished devices are again contaminated with PMMA residues caused by the metal lift-off with PMMA, but the goal of this experiment is to track the possible degradation of the electrical performance due to the initial hydrogen plasma clean.

Representative transfer characteristics of the shortest channel devices $(0.5 \mu \mathrm{m})$ are shown in Fig. 5(c), where the drain current density $I_{d}$ is plotted as a function of the back gate voltage $V_{g}$ at a fixed value of source-drain bias of $1 \mathrm{~V}$. Devices fabricated on pristine $W_{2}$ and on plasma exposed $W_{2}\left(6 \mathrm{~s}\right.$ at $\left.300^{\circ} \mathrm{C}\right)$ demonstrate comparable $I_{\text {on }} / I_{\text {off }}$ ratio of about $10^{7}$ and a maximum source-drain current of about $1 \mu \mathrm{A} / \mu \mathrm{m}$. This confirms the low-damage nature of remote $\mathrm{H}_{2}$ plasma cleaning. Since plasma treatment was performed before the contact pad deposition, both channel and contact regions of the transistors were affected by $\mathrm{H}_{2}$ plasma exposure.

In order to decouple the effect of $\mathrm{H}_{2}$ plasma on the channel and contact regions, a set of $\mathrm{WS}_{2}$ devices was fabricated and treated by $\mathrm{H}_{2}$ plasma only after contact lift-off. Therefore, only the channel region is exposed to the plasma and the contact region is protected by the metal. This leads to a severe degradation of device performance as shown in Fig. 5(c) (blue curve). The latter can be explained by the detrimental effect of acetone (used for contact lift-off) and $\mathrm{H}_{2}$ plasma already observed in PL measurements, as shown in Fig. 4. Therefore, for the remainder of the current paragraph, the attention was brought to samples which are plasma-treated before pad fabrication.
Figure 5(d, e) show $I_{\text {on }}$ and sub-threshold swing SS obtained for multiple identical devices per sample, plotted for various $\mathrm{H}_{2}$ plasma and resulfurization treatments. The channel length was chosen as $15 \mu \mathrm{m}$, as these are better probes for mobility degradation caused by plasma damage, contrary to short channel devices depicted in Fig. 5(c). As anticipated from the PL measurements shown in the previous paragraphs, the $\mathrm{H}_{2}$ plasma cleaning leads to one order of magnitude decrease in $I_{\text {on, }}$ which is indicative of a mobility degradation. Concomitantly, the subthreshold swing slope doubles, which is indicative of a higher amount of trapped charges and a loss of electrostatic control. This confirms that the $\mathrm{WS}_{2}$ is modified by the $\mathrm{H}^{*}$ flux, even at high temperature $\left(300^{\circ} \mathrm{C}\right)$ and short exposure time $(6 \mathrm{~s})$.

Performance recovery by resulfurization with ex situ $\mathrm{H}_{2} \mathrm{~S}$ annealing for temperatures ranging from 250 to $1000^{\circ} \mathrm{C}$ is studied with sample set 1 . Both $I_{\text {on }}$ and SS are fully restored by annealing at $1000^{\circ} \mathrm{C}$, while the restoration remains partial at lower temperature. This is consistent with synthesis studies by the metal-oxide sulfurization method, also requiring high temperature for optimal results ${ }^{34}$. The sample set 2 investigates in situ resulfurization by means of OCS soaking, in similar conditions as described earlier in this paper. Similarly as for sample set 1 , the application of $\mathrm{H}_{2}$ plasma cleaning alone causes $\mathrm{WS}_{2}$ degradation, with a strong decreased in $I_{\text {on }}$ and increase in SS. By performing a soak in OCS for 4 min directly after $\mathrm{H}_{2}$ DPS, without vacuum break, the electrical properties of the $\mathrm{WS}_{2}$ monolayer are almost fully restored, confirming the beneficial effect of this treatment as observed earlier by photoluminescence (see Fig. 2(c)).

In summary, remote hydrogen plasma can fully remove polymer layers from monolayer $\mathrm{WS}_{2}$ with only a minor degradation of its morphological, optical, chemical and electrical properties. This is 


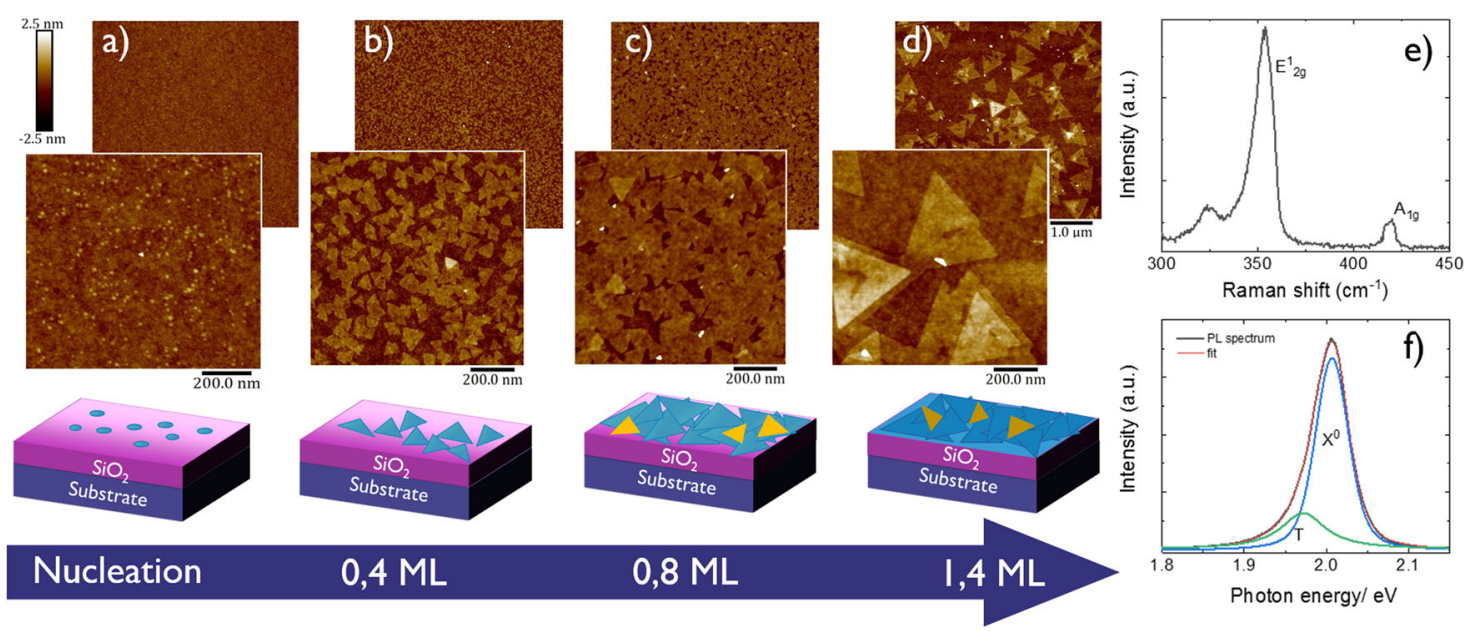

Fig. 6 Characterization of as-grown $\mathrm{WS}_{2}$ on $\mathrm{Si}_{\mathbf{S}} \mathrm{SiO}_{2}$ by MOCVD. a-d AFM images at various stages of the growth (the layer thickness in each step is given based on RBS analysis); e representative Raman spectrum of a representative monolayer sample; $\mathbf{f}$ photoluminescence (PL) measurements of a representative monolayer sample, showing neutral exciton (X0) and trion (T) contributions.

not possible with conventional wet or thermal treatments. In the downstream plasma source, at high substrate temperature PMMA is readily etched by reactive $\mathrm{H}$ atoms with an etch rate as high as $100 \mathrm{~nm} / \mathrm{s}\left(300^{\circ} \mathrm{C}\right)$. In contrast with the thermally activated PMMA etching, $\mathrm{WS}_{2}$ damage by atomic hydrogen is significantly reduced when the treatment temperature is increased. This unexpected temperature effect is explained by the specific mechanism of sulfur vacancy generation by atomic hydrogen, through the production of gas phase $\mathrm{H}_{2} \mathrm{~S}$ molecules. At low surface temperature, the coverage and the residence time of adsorbed $\mathrm{H}$ atoms on $\mathrm{WS}_{2}$ are sufficiently high so that $\mathrm{H}_{2} \mathrm{~S}_{\text {surf }}$ and $\mathrm{HS}_{\text {surf }}$ groups can form, promoting to the release of $\mathrm{H}_{2} \mathrm{~S}(\mathrm{~g})$. At elevated substrate temperature, $\mathrm{H}$ atoms quickly desorb, reducing the $\mathrm{H}_{2} \mathrm{~S}_{\text {surf }}$ and $\mathrm{HS}_{\text {surf }}$ surface concentrations. After low-damage hydrogen plasma cleaning, the residual sulfur depletion can be healed by soaking the samples, without vacuum break, for short time in a carbonyl sulfide (OCS) atmosphere. As demonstrated by DFT, OCS-based resulfurization results from the formation of an intermediate OCSH surface radical, which dissociate on the $\mathrm{S}$ vacancy, releasing a volatile $\mathrm{CO}$ molecule. The effect of $\mathrm{H}_{2}$ dry cleaning and in situ OCS restoration, observed on monolayer $\mathrm{WS}_{2}$ films, were confirmed on back-gated field-effect transistors, showing its advantages over high-temperature ex situ $\mathrm{H}_{2} \mathrm{~S}$ annealing.

The proposed PMMA dry cleaning technique can be implemented at moderate cost at lab level, for research activities involving the transfer and fabrication of small devices, using CVD material or flakes produced by exfoliation. From the large-scale manufacturing perspective, remote hydrogen plasma cleaning, combined with OCS soak, is fast and can be performed with existing fab equipment. Although the process performance has been demonstrated for the PMMA/WS $\mathrm{WS}_{2}$ combination we expect that plasma cleaning will be efficient for low-damage removal of various polymers from a broad range of $2 \mathrm{D}$ monolayers such as graphene, $\mathrm{MoS}_{2}$ and other TMDs.

\section{METHODS}

\section{Monolayer $\mathbf{W S}_{\mathbf{2}}$}

The $\mathrm{WS}_{2}$ films were grown directly on $300 \mathrm{~mm}$ silicon wafers covered with a $90 \mathrm{~nm}$ thermal oxide layer, in an upgraded MOCVD tool compatible with $300 \mathrm{~mm}$ CMOS flow. The film is a fully closed monolayer with multilayer islands as discussed below. The Fig. 6(a-d) illustrate various stages of the growth. As a first step, the wafer is heated under high-purity $\mathrm{N}_{2} / \mathrm{H}_{2}$ until it reaches $750^{\circ} \mathrm{C}$. The growth starts with a nucleation step, where $\mathrm{W}(\mathrm{CO})_{6}$ (partial pressure $\sim 9 \times 10^{-3}$ Torr) and $\mathrm{H}_{2} \mathrm{~S}$ are introduced (precursor partial pressures ratio $\mathrm{H}_{2} \mathrm{~S} / \mathrm{W}(\mathrm{CO})_{6}=5 \times 10^{3}$ ) for $10 \mathrm{~min}$ at $750^{\circ} \mathrm{C}$ and a chamber pressure of 150 Torr. This nucleation step induces arbitrary-oriented $\mathrm{WS}_{2}$ grains, with a density of $\sim 8 \times 10^{9} / \mathrm{cm}^{2}$ (Fig. $6(\mathrm{a})$ ). In order to enhance the lateral growth ${ }^{39,40}$ and ensure the closure of the first monolayer (ML), the chamber temperature is then increased to $900^{\circ} \mathrm{C}$ and the pressure decreased to 20 Torr (Fig. $6(\mathrm{~b}, \mathrm{c})$ ). It is, however, noticeable how secondary domains starts to grow even before the first monolayer fully coalesces (Fig. $6(c))$, which is in-line with the model predictions ${ }^{41}$. The growth then continues until the first ML growth is fully closed, in addition to subsequent multilayer grains on arbitrary location.

Figure 6(d) shows an AFM image of pristine $\mathrm{WS}_{2}$ indicating the presence of a fully closed monolayer with triangular multilayer patches. Raman and photoluminescence (PL) measurements confirm that $\mathrm{WS}_{2}$ is predominantly monolayer (see Fig. $6(\mathrm{e}, \mathrm{f})$ ). In Fig. 6(f), the PL spectrum is fitted with two Voigt peak functions corresponding to neutral exciton (centered at $2.00 \mathrm{eV}$ ) and trion $(1.97 \mathrm{eV})$ emission in agreement with literature data ${ }^{14,42,43}$. The spectral width of the PL peak is about $50 \mathrm{meV}$ and the intensity is two orders of magnitude higher than the Raman $\mathrm{A}_{1 \mathrm{~g}}$ peak. This indicates the high crystalline quality of the MOCVD material is comparable to state of the art synthetic ${ }^{44}$ and exfoliated ${ }^{45} W_{2}$. Moreover, excellent $\mathrm{WS}_{2}$ uniformity across a $300 \mathrm{~mm}$ substrate was confirmed by PL and AFM mappings.

\section{Plasma cleaning, in situ, and ex situ restoration}

In this study, a downstream plasma source (DPS) from Tokyo Electron Limited (TEL), operating in $\mathrm{Ar} / \mathrm{H}_{2}$ mixtures, was used. This plasma system was attached to a TEL Tactras ${ }^{\mathrm{TM}}$ platform with six available slots, among which a Vesta NV TM chamber was attached, which was used for OCS soak. In DPS, energetic hydrogen ions and UV radiation from the plasma are effectively blocked by a set of baffles allowing only reactive thermal $\mathrm{H}$ atoms to interact with the treated substrate. The temperature of the wafer holder can be varied between 60 and $300^{\circ} \mathrm{C}$ by resistive heaters. Fixed DPS settings $\left(\mathrm{Ar} / \mathrm{H}_{2}=1000 / 40 \mathrm{sccm}, P_{\mathrm{RF}}=500 \mathrm{~W}, p=1\right.$ Torr) were employed throughout this study, only the duration of plasma exposure and the temperature of the substrate were varied. The system is capable of handling full $300 \mathrm{~mm}$ wafers, and/or smaller samples by means of a Silicon pocket wafer. Before any processing, the chamber was conditioned for 10 min with a high power/high flux $\mathrm{Ar} / \mathrm{H}_{2}$ discharge in order to remove any residual trace of oxygen or fluorine. Samples used for the current study had dimensions of $1 \times 1 \mathrm{~cm}^{2}$ and were cleaved out of $300 \mathrm{~mm} \mathrm{WS}$ wafers, deposited by MOCVD (see above). The coupons were placed in the center of a $300 \mathrm{~mm}$ carrier Si wafer equipped with a central pocket avoiding sliding out of the sample during robot transportation in the Tactras ${ }^{\mathrm{TM}}$ platform. In order to ensure proper thermal stability of the samples while processed in the DPS chamber, after placing the Si carrier on the hot table, the temperature was stabilized for 2 min before turning on the plasma source. The DPS chamber was connected through a vacuum transport module (purged with $\mathrm{N}_{2}, 90$ mTorr), to the soaking Vesta $\mathrm{NV}^{\mathrm{TM}}$ chamber, where the wafer was exposed to carbonyl sulfide for $4 \mathrm{~min}$ at a pressure of $100 \mathrm{mTorr}$ and a substrates temperature of $60^{\circ} \mathrm{C}$. The ex situ $\mathrm{H}_{2} \mathrm{~S}$ annealing 
were performed in a $300 \mathrm{~mm}$ Eagle12 (Polygon 8300) ALD system from ASM, compatible with Si pocket wafers for the treatment of small size samples. The treatment time was 10 min under a $\mathrm{H}_{2} \mathrm{~S}$ pressure of 20 Torr.

\section{PMMA removal}

In order to investigate PMMA removal, a $200 \mathrm{~nm}$ thick PMMA layer (Allresist, molecular weight $200 \mathrm{~K}, 3 \%$ anisole solution) was spin-coated on as-grown $\mathrm{WS}_{2}$ followed by a $3 \mathrm{~min}$ bake at $165^{\circ} \mathrm{C}$. To benchmark our downstream plasma cleaning against the conventional acetone strip, PMMA-coated $\mathrm{WS}_{2}$ samples were cleaned in acetone overnight (16 h) at room temperature followed by isopropyl alcohol (IPA) rinse and nitrogen drying. Bulk PMMA etch rate in the downstream $\mathrm{Ar} / \mathrm{H}_{2}$ plasma was measured on silicon samples coated with $\sim 200 \mathrm{~nm}$ or $\sim 600 \mathrm{~nm}$ of PMMA. The thickness of the polymer film before and after the etch was measured by spectroscopic ellipsometry (SE).

\section{Determination of the irreversibly adsorbed PMMA layer}

After native oxide measurement by spectroscopic ellipsometry, bare $\mathrm{Si}$ substrates are pre-annealed (removal adsorbed gasses) for $2 \mathrm{~min}$ at $135^{\circ} \mathrm{C}$; then spin-coated with PMMA (Allresist, molecular weight $600 \mathrm{~K}$, $4 \%$ anisole solution). Post-bake is performed under ambient atmosphere on a hot plate at various temperatures above $T_{\mathrm{g}}$ for various times. Afterwards, samples are dipped in acetone overnight (16h), then measured by SE to determine the thickness of the residual PMMA film; it was checked that the residual thickness remains constant for longer immersion times).

\section{Physical and chemical characteristics}

AFM images were acquired in tapping-mode in ambient conditions. A special procedure was developed to assess the presence of PMMA residues on the WS $\mathrm{Wurface}_{2}$. First, a small area (typically $2 \times 2 \mu \mathrm{m}^{2}$ ) is mechanically cleaned by performing several contact mode AFM scans as discussed in ref. ${ }^{46}$. Subsequent tapping-mode inspection of a larger area provides a clear visualization of the residue accumulation at the edges of the mechanically cleaned zone. Moreover, the height difference between mechanically cleaned and intact areas provides a direct measure of the thickness of the residual layer. The Raman spectroscopy and photoluminescence $(\mathrm{PL})$ measurements were performed using a Horiba JobinYvon HR800 instrument with a $532 \mathrm{~nm}$ laser excitation. The excitation beam was focused on the $\mathrm{WS}_{2}$ surface using a $100 \times$ objective with a spot size of $1 \mu \mathrm{m}$. Both Raman and PL measurements were performed at room temperature in the ambient environment. Chemical analysis of $W_{2}$ was done by XPS. The measurements were carried out in Angle Integrated mode using a Theta300 system from Thermolnstruments. A monochromatized Al Ka X-ray source $(1486.6 \mathrm{eV})$ was used with the spot size of $400 \mu \mathrm{m}$. The energy calibrations were made against the C1s peak to compensate for charging effects during analysis. To limit $\mathrm{WS}_{2}$ oxidation in the ambient environment the delay between the treatment and the XPS measurements was kept below $60 \mathrm{~min}$. The $\mathrm{WS}_{2}$ surface hydrophilicity, after surface treatment, was assessed by water contact angle (WCA) goniometry. The static WCA of deionized water droplets (volume of $2 \mu \mathrm{L}$ ), dispensed on the sample surface, was measured in air using a softwarecontrolled Video Contact Angle System from Dataphysics company. Transmission electron microscopy images were performed using a Talos system from FEl, operated at $200 \mathrm{kV}$ and equipped with SuperTwin FEI EDS detector; after spin-coating with a carbon layer, the lamellae were prepared by Focused Ion Beam (FIB) with a Helios G4 system.

\section{Patterned samples for electrical measurements}

Back-gated field-effect transistors were fabricated as follows. The $1 \mathrm{ML} \mathrm{WS}_{2}$ channel was grown by MOCVD on a $300 \mathrm{~mm} \mathrm{p+-doped} \mathrm{Si} \mathrm{wafer,} \mathrm{with}$ $50 \mathrm{~nm}$ thermal $\mathrm{SiO}_{2}$ as back gate oxide. The wafer was cleaved in pieces, treated with $\mathrm{H}$ remote plasma then various sulfur restoration treatments. Afterwards, contact pads were patterned by PMMA spin-coating, lithography by e-beam exposure, followed by solvent development, metallization with $10 \mathrm{~nm} \mathrm{Ni} / 20 \mathrm{~nm} \mathrm{Pd}$, and metal lift-off in acetone. The contact size was $104 \mu \mathrm{m}$ width and $80 \mu \mathrm{m}$ length, and the distance between two contacts, defining the channel length, varied from $500 \mathrm{~nm}$ to $15 \mu \mathrm{m}$. The individual devices were then electrically isolated by mechanical scratching of the surrounding $\mathrm{WS}_{2}$ with probe needles. Electrical measurements were done at room temperature using a Cascade PA300 probe station and a Keysight B1500A parameter analyzer. The back gate voltage was swept using the chuck contact, the drain pad was biased at 1 Volt and the source was grounded. The chamber was flushed with nitrogen to limit adsorption of moisture on the bare $\mathrm{WS}_{2}$ channel during the measurements.

Two metrics are considered to track the effect of $\mathrm{H}$ remote plasma and subsequent anneals on the material quality, the sub-threshold swing (SS), and the on-state current $\left(I_{o n}\right)$. The SS probes the amount of defects charging/discharging during back gate voltage sweeping and screening the electrostatic potential in the channel. The SS was extracted at a fixed current of $10^{-10} \mathrm{~A} / \mathrm{\mu m}^{2}$. The $I_{\text {on }}$ probes the amount of scattering centers degrading the electron mobility, and was extracted at the threshold voltage $\left(V_{t}\right)+10 \mathrm{~V}$ overdrive. This large overdrive is needed due to the thick $50 \mathrm{~nm} \mathrm{SiO}_{2}$ back gate oxide. $V_{\mathrm{t}}$ was obtained by extrapolating the linear current at peak transconductance to zero.

\section{DATA AVAILABILITY}

The data that support the findings of this study are available from the corresponding author upon reasonable request.

\section{CODE AVAILABILITY}

The codes that support the findings of this study, detailed in the Supplementary Discussion, are available from the corresponding author upon reasonable agreement.

Received: 7 August 2020; Accepted: 12 December 2020; Published online: 28 January 2021

\section{REFERENCES}

1. Akinwande, D. et al. Graphene and two-dimensional materials for Silicon technology. Nature 573, 507-518 (2019).

2. Chhowalla, M., Jena, D. \& Zhang, H. Two-dimensional semiconductors for transistors. Nat. Rev. Mater. 1, 16052 (2016).

3. Resta, G. V. et al. Doping-free complementary logic gates enabled by twodimensional polarity-controllable transistors. ACS Nano 12, 7039-7047 (2018).

4. Chiappe, D. et al. Controlled sulfurization process for the synthesis of large area $\mathrm{MoS}_{2}$ films and $\mathrm{MoS}_{2} / \mathrm{WS}_{2}$ heterostructures. Adv. Mater. Interf. 3, 1500635 (2016).

5. Heyne, M. H. et al. The conversion mechanism of amorphous silicon to stoichiometric $\mathrm{WS}_{2}$. J. Mater. Chem. C 6, 4122-4130 (2018).

6. Heyne, M. H., de Marneffe, J.-F., Radu, I., Neyts, E. C. \& De Gendt, S. Thermal recrystallization of short-range ordered $W_{2}$ films. J. Vac. Sci. Technol. A 36, $05 G 501$ (2018).

7. Groven, B. et al. Plasma-enhanced atomic layer deposition of two-dimensional $W_{2}$ from $W_{6}, H_{2}$ plasma, and $\mathrm{H}_{2} \mathrm{~S}$. Chem. Mater. 29, 2927-2938 (2017).

8. Groven, B. et al. Nucleation mechanism during $\mathrm{WS}_{2}$ plasma enhanced atomic layer deposition on amorphous $\mathrm{Al}_{2} \mathrm{O}_{3}$ and sapphire substrates. J. Vac. Sci. Technol. A 36, $01 \mathrm{~A} 105$ (2018).

9. van der Zande, A. M. et al. Grains and grain boundaries in highly crystalline monolayer molybdenum disulphide. Nat. Mater. 12, 554-561 (2013).

10. Kang, K. et al. High-mobility three-atom-thick semiconducting films with waferscale homogeneity. Nature 520, 656-660 (2015).

11. Lin, Y.-C. et al. Graphene annealing: How clean can it be? Nano Lett. 12, 414-419 (2012).

12. Cheng, Z. et al. Toward intrinsic graphene surfaces: a systematic study on thermal annealing and wet-chemical treatment of $\mathrm{SiO}_{2}$-supported graphene devices. Nano Lett. 11, 767-771 (2011).

13. Cunge, G. et al. Dry efficient cleaning of poly-methyl-methacrylate residues from graphene with high-density $\mathrm{H}_{2}$ and $\mathrm{H}_{2}-\mathrm{N}_{2}$ plasmas. J. Appl. Phys. 118, 123302 (2015).

14. McCreary, K. M. et al. The effect of preparation conditions on raman and photoluminescence of monolayer WS $\mathrm{WS}_{2}$ Sci. Rep. 6, 35154 (2016).

15. Donarelli, M., Bisti, F., Perrozzi, F. \& Ottaviano, L. Tunable sulfur desorption in exfoliated $\mathrm{MoS}_{2}$ by means of thermal annealing in ultra-high vacuum. Chem. Phys. Lett. 588, 198-202 (2013).

16. Heyne, $M$. H. et al. A route towards the fabrication of $2 D$ heterostructures using atomic layer etching combined with selective conversion. 2D Materials 6, 035030 (2019).

17. Wood, J. D. et al. Annealing free, clean graphene transfer using alternative polymer scaffolds. Nanotechnology 26, 055302 (2015).

18. Yamamoto, M., Maruoka, T., Kono, A., Horibe, H. \& Umemoto, H. Substrate temperature dependence of the photoresist removal rate using atomic hydrogen generated by a hot-wire tungsten catalyst. Jpn. J. Appl. Phys. 49, 016701 (2010).

19. Umemoto, H., Kato, T., Takiguchi, M., Takagi, S. \& Horibe, H. Decomposition processes of photoresist polymers by $\mathrm{H}$ atoms produced on hot wire surfaces. Thin Solid Films 635, 27-31 (2017). 
20. Cheng, C.-C. et al. Activating basal-plane catalytic activity of two-dimensional $\mathrm{MoS}_{2}$ monolayer with remote hydrogen plasma. Nano Energy 30, 846-852 (2016).

21. Lu, A.-Y. et al. Janus monolayers of transition metal dichalcogenides. Nat. Nanotechnol. 12, 744-749 (2017).

22. Sorescu, D. C., Sholl, D. S. \& Cugini, A. V. Density functional theory studies of the interaction of $\mathrm{H}, \mathrm{S}, \mathrm{Ni}-\mathrm{H}$, and Ni-S complexes with the MoS2 basal plane. J. Phys. Chem. B 108, 239-249 (2004).

23. Huyghebaert, C. et al. 2D materials: roadmap to CMOS integration. In 2018 IEEE International Electron Devices Meeting (IEDM), 22.1.1-22.1.4 (IEEE, 2018).

24. Guiselin, O. Irreversible adsorption of a concentrated polymer solution. Europhys. Lett. (EPL) 17, 225-230 (1992).

25. Napolitano, S. Irreversible adsorption of polymer melts and nanoconfinement effects. Soft Matter 16, 5348-5365 (2020).

26. van der Lee, A., Hamon, L., Holl, Y. \& Grohens, Y. Density profiles in thin PMMA supported films investigated by X-ray reflectometry. Langmuir 17, 7664-7669 (2001).

27. Simavilla, D. N. et al. Mechanisms of polymer adsorption onto solid substrates. ACS Macro Lett. 6, 975-979 (2017).

28. Housmans, C., Sferrazza, M. \& Napolitano, S. Kinetics of irreversible chain adsorption. Macromolecules 47, 3390-3393 (2014).

29. Ohrt, C. et al. Free volume profiles at polymer-solid interfaces probed by focused slow positron beam. Macromolecules 48, 1493-1498 (2015).

30. Budd, P. M., McKeown, N. B. \& Fritsch, D. Free volume and intrinsic microporosity in polymers. J. Mater. Chem. 15, 1977-1986 (2005).

31. Leng, C. Z. \& Losego, M. D. Vapor phase infiltration (VPI) for transforming polymers into organic-inorganic hybrid materials: a critical review of current progress and future challenges. Mater. Horiz. 4, 747-771 (2017).

32. Rosenberger, M. R., Chuang, H.-J., McCreary, K. M., Li, C. H. \& Jonker, B. T. Electrical characterization of discrete defects and impact of defect density on photoluminescence in monolayer WS 2 . ACS Nano 12, 1793-1800 (2018).

33. Carozo, V. et al. Optical identification of sulfur vacancies: Bound excitons at the edges of monolayer tungsten disulfide. Sci. Adv. 3, e1602813 (2017).

34. Heyne, M. H. et al. The conversion mechanism of amorphous silicon to stoichiometric $W_{2}$. J. Mater. Chem. C 6, 4122-4130 (2018).

35. Perini, C. J., Muller, M. J., Wagner, B. K. \& Vogel, E. M. Low-temperature, plasma assisted, cyclic synthesis of MoS $_{2}$. J. Vac. Sci. Technol. B 36, 031201 (2018).

36. Jeon, $\mathrm{M}$. H. et al. Controlled $\operatorname{mos}_{2}$ layer etching using $\mathrm{CF}_{4}$ plasma. Nanotechnology 26, 355706 (2015)

37. Kim, J. K. et al. Study on the etching characteristics of amorphous carbon layer in oxygen plasma with carbonyl sulfide. J. Vac. Sci. Technol. A 31, 021301 (2013).

38. Voronina, E. N. et al. Mechanisms of hydrogen atom interactions with $\mathrm{MoS}_{2}$ monolayer. J Phys.: Condensed Matter 32, 445003 (2020).

39. Zhang, X. et al. Diffusion-controlled epitaxy of large area coalesced $\mathrm{WSe}_{2}$ monolayers on sapphire. Nano Lett. 18, 1049-1056 (2018).

40. Eichfeld, S. M., Colon, V. O., Nie, Y., Cho, K. \& Robinson, J. A. Controlling nucleation of monolayer $\mathrm{WSe}_{2}$ during metal-organic chemical vapor deposition growth. $2 D$ Materials 3, 025015 (2016).

41. $\mathrm{Ye}, \mathrm{H}$. et al. Toward a mechanistic understanding of vertical growth of van der waals stacked 2D materials: a multiscale model and experiments. ACS Nano 11, 12780-12788 (2017).

42. Jadczak, J. et al. Probing of free and localized excitons and trions in atomically thin $\mathrm{WSe}_{2}, \mathrm{WS}_{2}, \mathrm{MoSe}_{2}$ and $\mathrm{MoS}_{2}$ in photoluminescence and reflectivity experiments. Nanotechnology 28, 395702 (2017).

43. Currie, M., Hanbicki, A. T., Kioseoglou, G. \& Jonker, B. T. Optical control of charged exciton states in tungsten disulfide. Appl. Phys. Lett. 106, 201907 (2015).

44. Gutiérrez, H. R. et al. Extraordinary room-temperature photoluminescence in triangular WS 2 monolayers. Nano Lett. 13, 3447-3454 (2013).

45. Cadiz, F. et al. Excitonic linewidth approaching the homogeneous limit in $\mathrm{MoS}_{2}-$ based van der waals heterostructures. Phys. Rev. X 7, 021026 (2017).

46. Goossens, A. M. et al. Mechanical cleaning of graphene. Appl. Phys. Lett. 100, 073110 (2012)

\section{ACKNOWLEDGEMENTS}

Daniil Marinov has received funding from the European Union's Horizon 2020 research and innovation program under the Marie Sklodowska-Curie grant agreement No 752164. Ekaterina Voronina, Yuri Mankelevitch, and Tatyana Rakhimova are thankful to the Russian Science Foundation (RSF) for financial support (Grant No. 16-12-10361). This study was carried out using the equipment of the shared research facilities of high-performance computing resources at Lomonosov Moscow State University and the computational resources and services of the HPC core facility CalcUA of the University of Antwerp, and VSC (Flemish Supercomputer Center), funded by the Research Foundation-Flanders (FWO) and the Flemish Government. Patrick With gratefully acknowledges imec's CTO office for financial support during his stay at imec. The authors thank Mr. Surajit Sutar (imec) for his help during sample electrical characterization, and Patrick Verdonck for lab processing. Jean-François de Marneffe thank Prof. Simone Napolitano from the Free University of Brussels for useful discussions on irreversibly adsorbed polymer layers, and Cédric Huyghebaert (imec) for his continuous support in the framework of the Graphene FET Flagship core project. All authors acknowledge the support of imec's pilot line and materials characterization and analysis (MCA) group, namely Jonathan Ludwig, Stefanie Sergeant, Thomas Nuytten, Olivier Richard, and Thierry Conard. Finally, Daniil Marinov thank Mikhail Krishtab (imec/KU Leuven) for his help in selecting the optimal plasma etch system for this work. Part of this project has received funding from the European Union's Horizon 2020 research and innovation program under grant agreement No 649953.

\section{AUTHOR CONTRIBUTIONS}

The manuscript was written by D.M. and J.-F.d.M. with contributions from S.E.K. and Q.S.. The manuscript was revised by all co-authors. All authors have given approval to the final version of the manuscript. D.M., with support from J.-F.d.M., performed plasma patterning tests and coordinated the sample's physical and chemical characterization. The $\mathrm{WS}_{2}$ samples were grown by S.E.K.. The initial $\mathrm{H}_{2}$ cleaning experiments were performed by M.H.H. and J.Z. The in-depth characterization and understanding of the PMMA removal mechanism were performed by J.Z. and P.-J.W. P.C.W. helped with the interpretation of XPS spectra and performed water contact angle measurements (wetting) at various stages of the cleaning treatments (not shown in the paper). The characterization by transmission electron microscopy (TEM) was performed by A.N.M. Q.S. coordinated and executed the work on the patterned electrical vehicles, helped by G.A. and S.B. K.B. performed modeling at university of Antwerp, under the direction of Prof. Erik Neyts, while E.V. performed simulations at Moscow State University, assisted by T.R. and Y.M. J.-F.d.M., I.A., and D.L. managed the overall activities, at the interface between imec's beyond-CMOS program and Unit Process Modules department. D.M. and J.-F.d.M. were advised by Prof. S.D.G. from imec v.z.w. and KU Leuven.

\section{COMPETING INTERESTS}

The authors declare no competing interests.

\section{ADDITIONAL INFORMATION}

Supplementary information is available for this paper at https://doi.org/10.1038/ s41699-020-00197-7.

Correspondence and requests for materials should be addressed to J.-F.d.M.

Reprints and permission information is available at http://www.nature.com/ reprints

Publisher's note Springer Nature remains neutral with regard to jurisdictional claims in published maps and institutional affiliations.

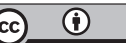

Open Access This article is licensed under a Creative Commons Attribution 4.0 International License, which permits use, sharing, adaptation, distribution and reproduction in any medium or format, as long as you give appropriate credit to the original author(s) and the source, provide a link to the Creative Commons license, and indicate if changes were made. The images or other third party material in this article are included in the article's Creative Commons license, unless indicated otherwise in a credit line to the material. If material is not included in the article's Creative Commons license and your intended use is not permitted by statutory regulation or exceeds the permitted use, you will need to obtain permission directly from the copyright holder. To view a copy of this license, visit http://creativecommons. org/licenses/by/4.0/.

(c) The Author(s) 2021 